\title{
Microwave-Assisted Rapid Synthesis of Nanoscale MOF-303 for Hydrogel Composites with Superior Proton Conduction at Ambient Humid Condition
}

Ya-Ru Kong, ${ }^{a}$ Ru Zhang, ${ }^{a}$ Jin Zhang, ${ }^{a}$ Hong-Bin Luo, ${ }^{* a}$ Yangyang Liu, ${ }^{b}$ Yang Zou, ${ }^{a}$ Xiao-Ming Ren*a,c


Chemistry and Molecular Engineering, Nanjing Tech University, Nanjing 211816, P.

R. China

b Department of Chemistry and Biochemistry, California State University, Los Angeles, 5151 State University Drive, Los Angeles, CA 90032-8202, USA

c State Key Laboratory of Coordination Chemistry, Nanjing University, Nanjing 210023, P. R. China

Tel: $86-25-58139476$

E-mail: hbluo@njtech.edu.cn (HBL); xmren@njtech.edu.cn (XMR) 


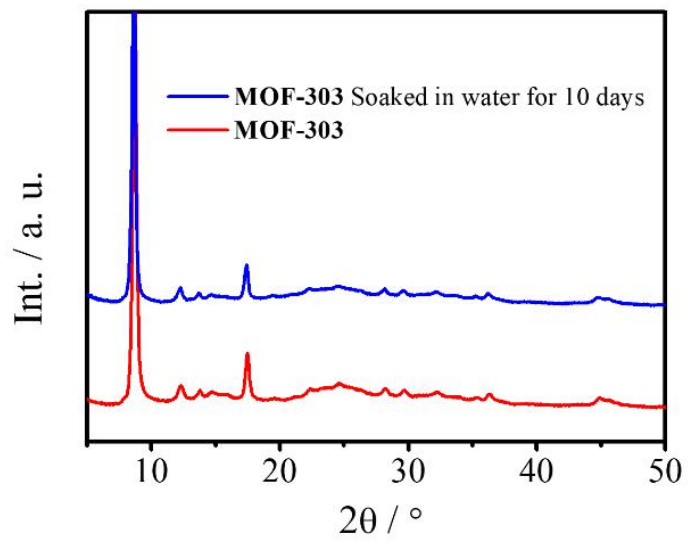

Figure S1. PXRD patterns of MOF-303 and MOF-303 immersed in water for 10 days.
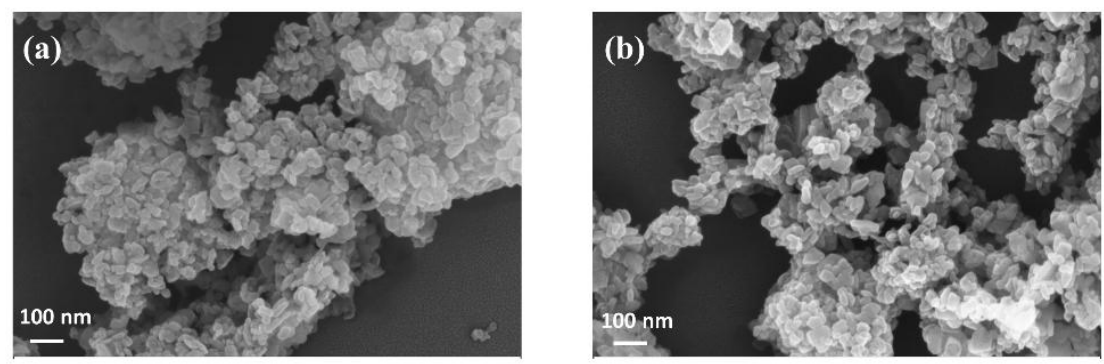

Figure S2. SEM images of (a) MOF-303 and (b) MeSA@MOF-303.
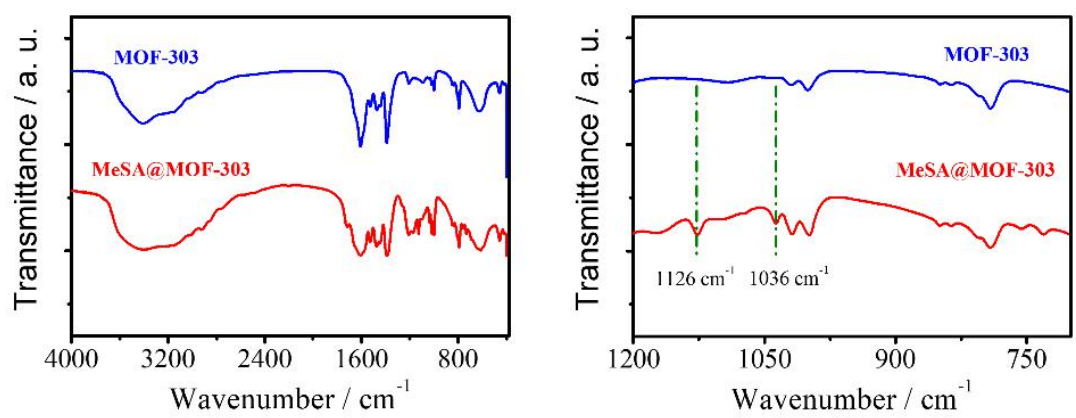

Figure S3. FT-IR spectra of MOF-303 and MeSA@MOF-303. 


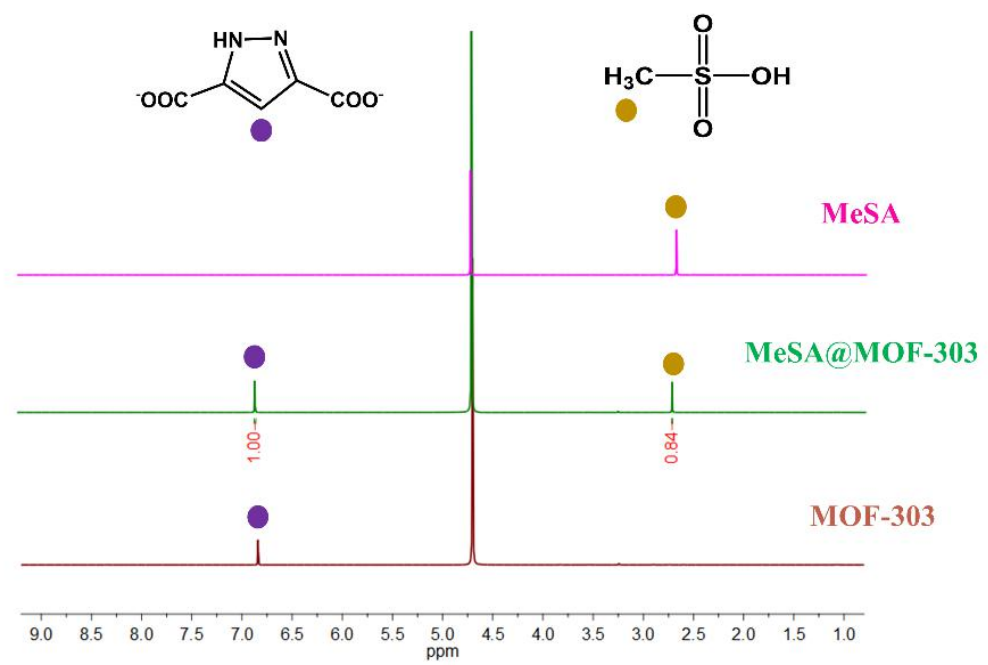

Figure S4. ${ }^{1} \mathrm{H}$ NMR spectra of MeSA, digested MeSA@MOF-303 and MOF-303 in $\mathrm{KOH} / \mathrm{D}_{2} \mathrm{O}$.

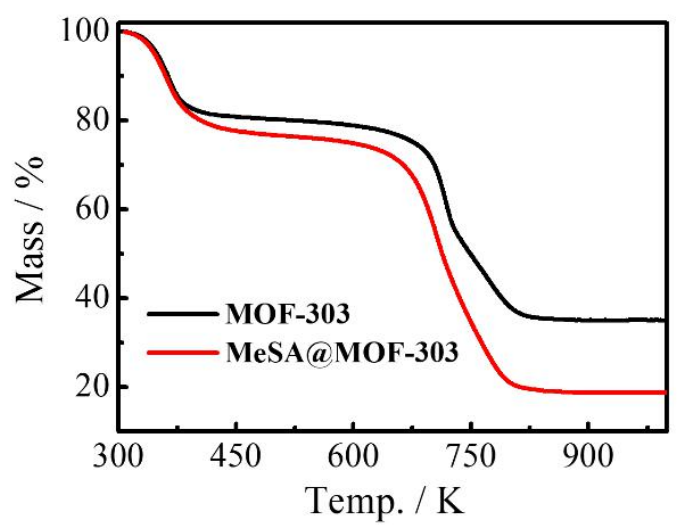

Figure S5. TG curves of MOF-303 and MeSA@MOF-303.

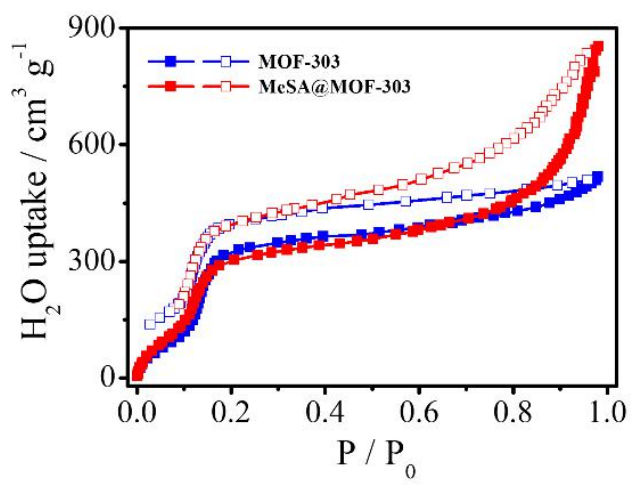

Figure S6. Water adsorption/desorption isotherms of MOF-303 and MeSA@MOF-303. 


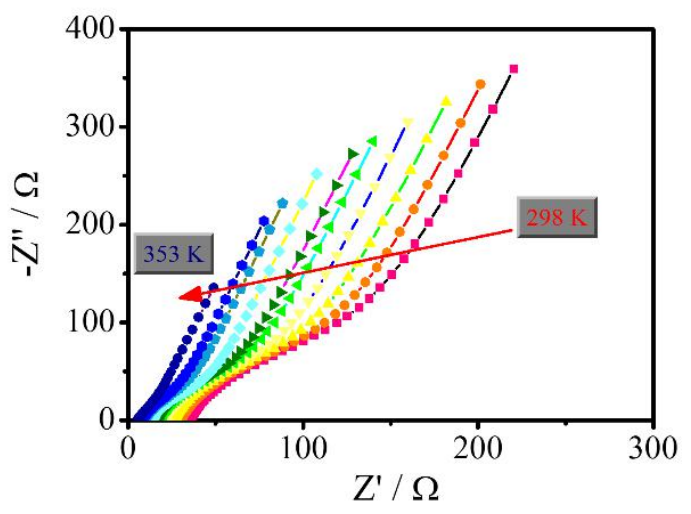

Figure S7. Temperature-dependent Nyquist plots of MeSA@MOF-303 under 98\% RH.

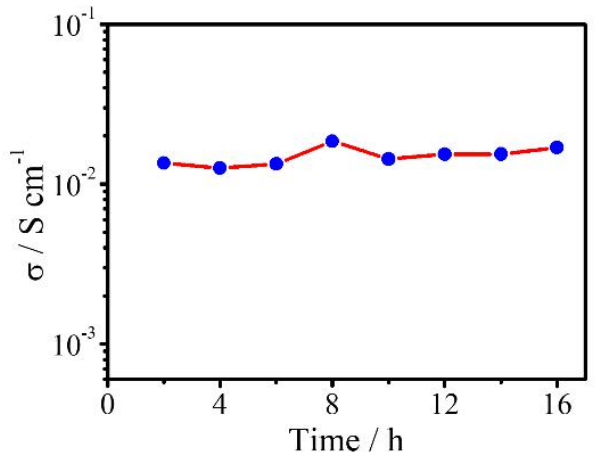

Figure S8. Proton-conducting long-term stability of MeSA@MOF-303 at $323 \mathrm{~K}$ and $98 \% \mathrm{RH}$.
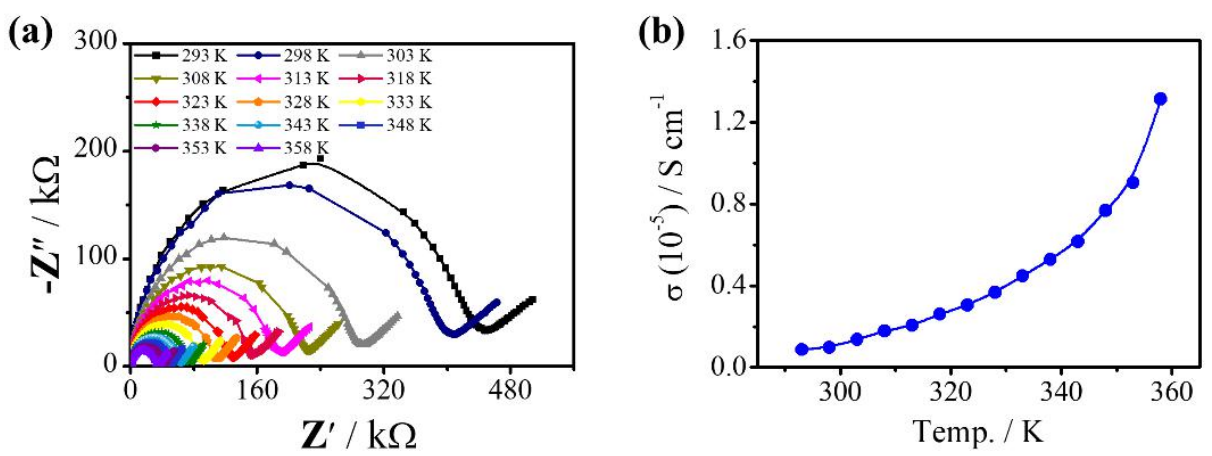

Figure S9. (a) Temperature-dependent Nyquist plots of MOF-303 under 98\% RH and (b) the corresponding proton conductivity. 

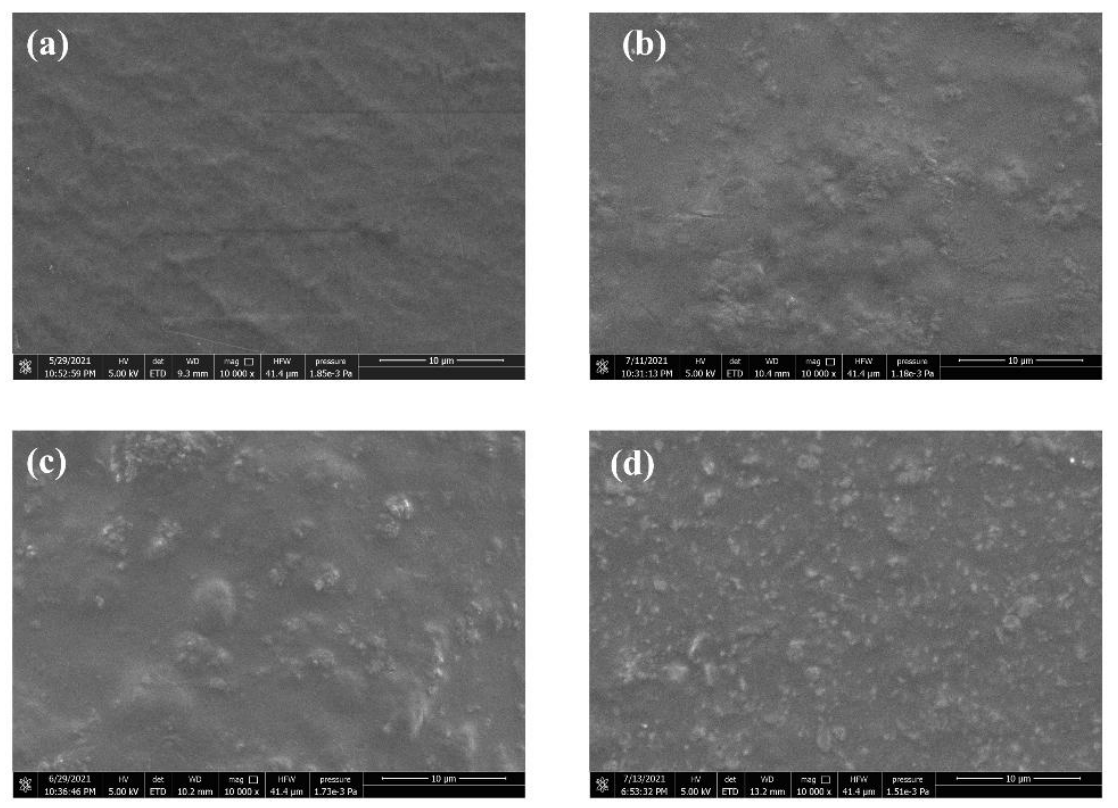

Figure S10. SEM images of (a) PVA hydrogel, (b) MeSA@MOF-303-PVA-25, (c)

MeSA@MOF-303-PVA-40, (d) MeSA@MOF-303-PVA-50.

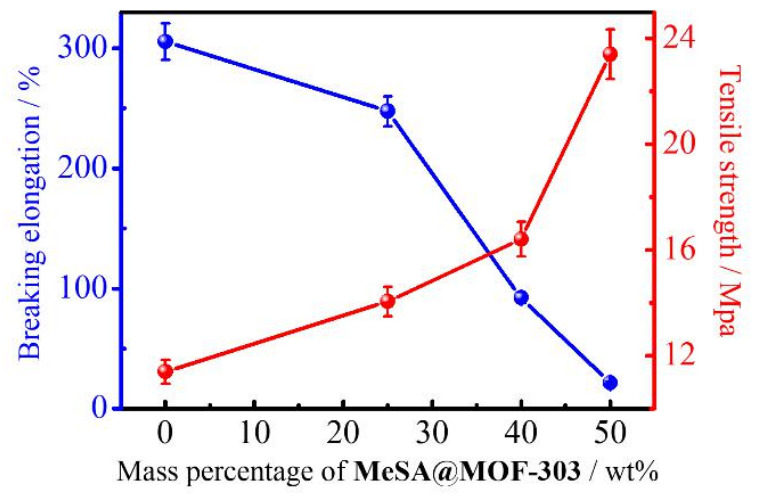

Figure S11. Plots of tensile strength and elongation at break against the masspercentage of MeSA@MOF-303 in the hydrogel composites. 

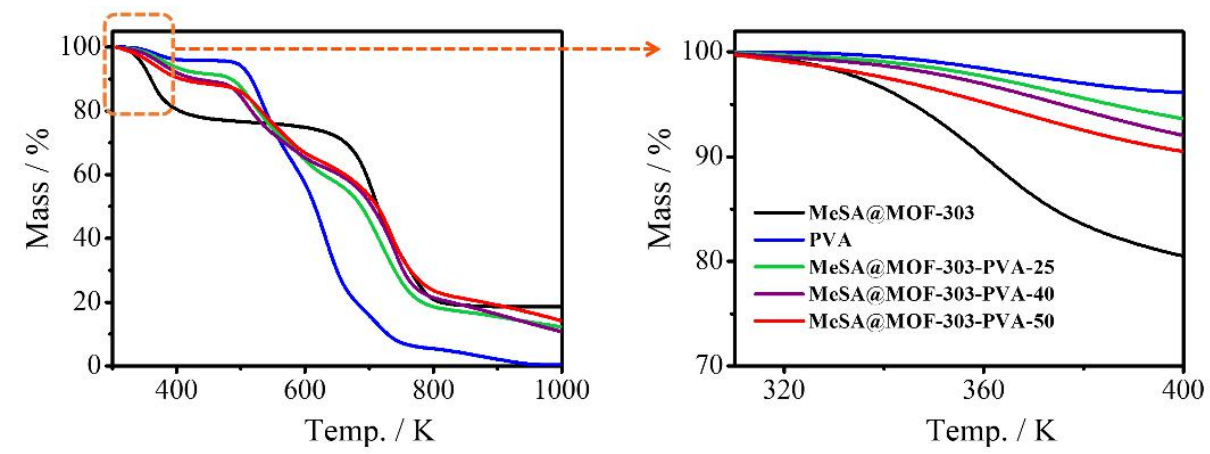

Figure S12. TG curves of MeSA@MOF-303, PVA hydrogel and

MeSA@MOF-303-PVA hydrogel composites.

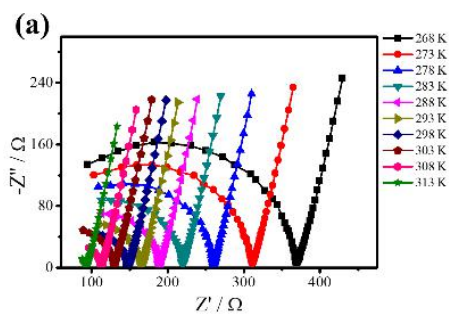

(b)

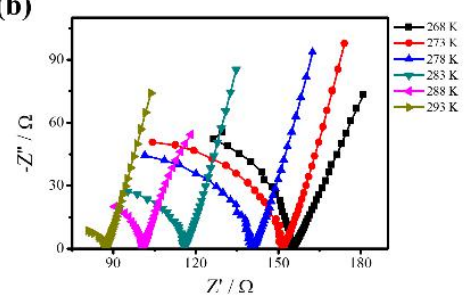

(e)

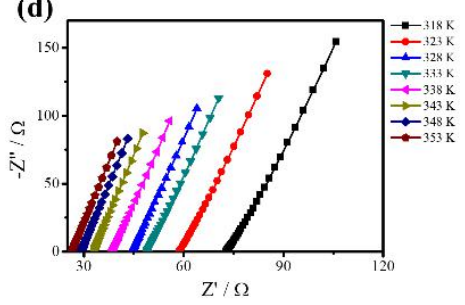

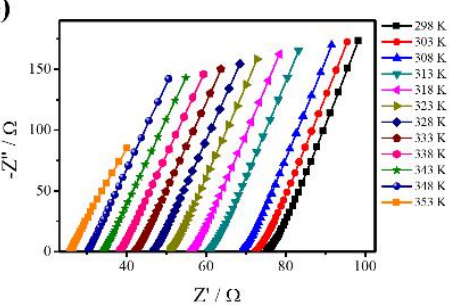

(c)
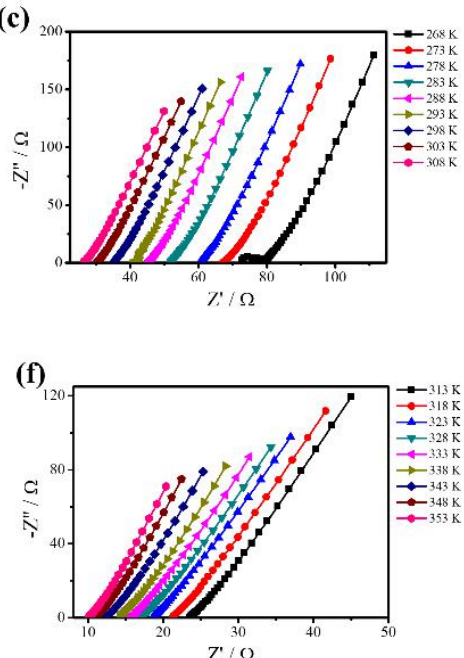

Figure S13. Temperature-dependent Nyquist plots at ambient humid condition for the hydrogel composites (a, d) MeSA@MOF-303-PVA-25, (b, e) MeSA@MOF-303-PVA-40 and (e, f) MeSA@MOF-303-PVA-50. 

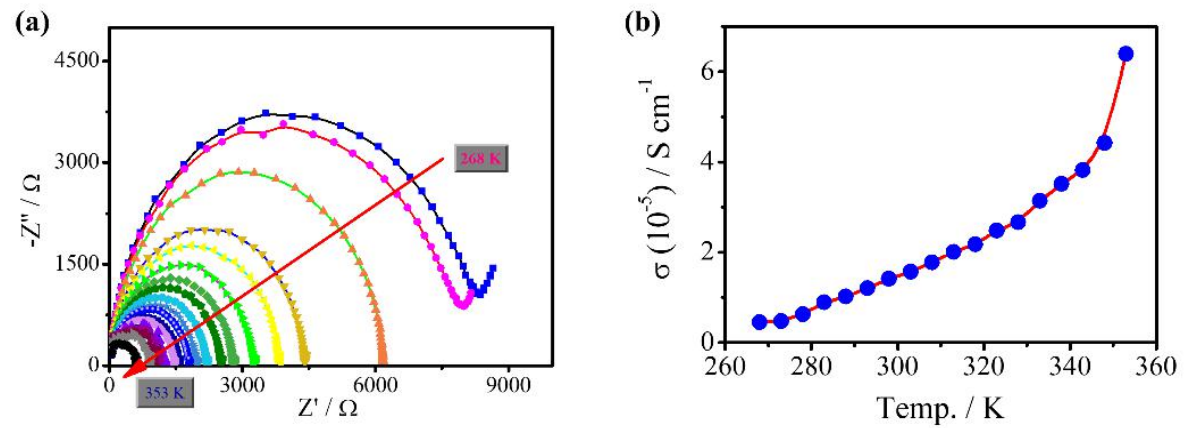

Figure S14. (a) Temperature-dependent Nyquist plots of PVA hydrogel at ambient humid condition and (b) the corresponding proton conductivity.

(a)

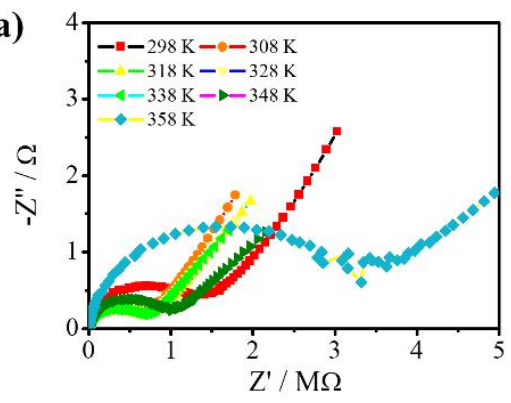

(b)

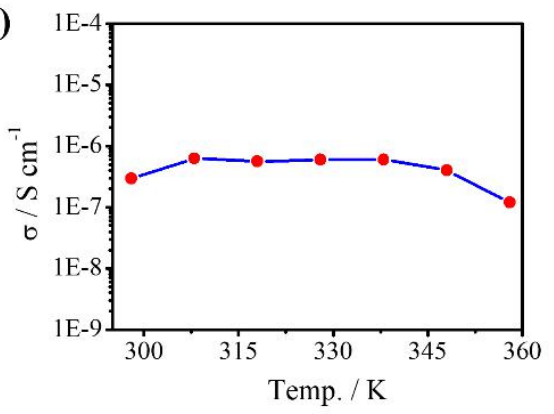

Figure S15. (a) Nyquist plots at varied temperatures and ambient humid condition for MeSA@MOF-303 and (b) the corresponding proton conductivities.
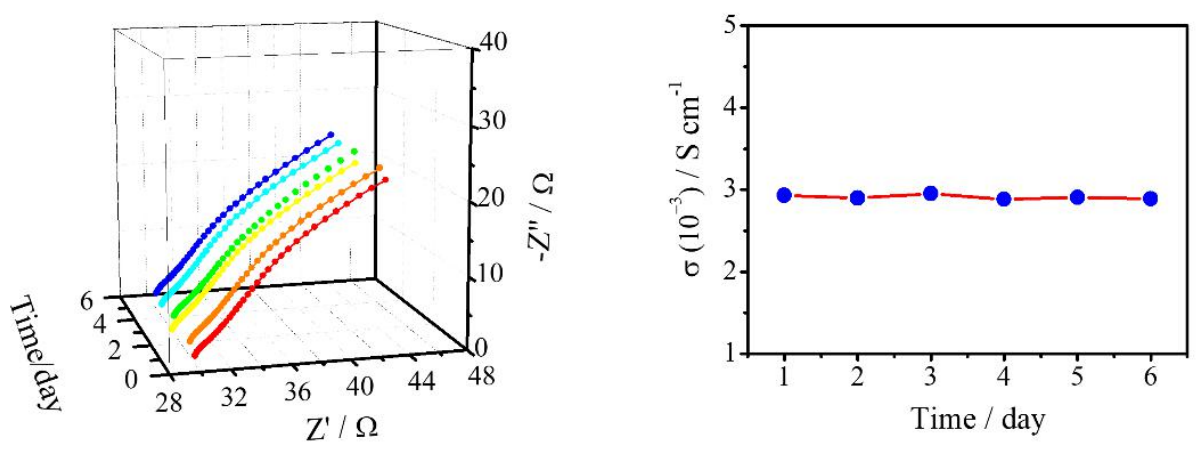

Figure S16. The long-term stability of MeSA@MOF-303-PVA-50 at room temperature and ambient humid condition. 